\title{
A deep sequencing reveals significant diversity among dominant variants and evolutionary dynamics of avian leukosis viruses in two infectious ecosystems
}

\author{
Fanfeng Meng ${ }^{1+}$, Xuan Dong ${ }^{1+}$, Tao $\mathrm{Hu}^{2}$, Shuang Chang ${ }^{1}$, Jianhua Fan ${ }^{3}$, Peng Zhao ${ }^{1 *}$ and Zhizhong Cui ${ }^{{ }^{*}}$
}

\begin{abstract}
Background: As a typical retrovirus, the evolution of Avian leukosis virus subgroup J (ALV-J) in different infectious ecosystems is not characterized, what we know is there are a cloud of diverse variants, namely quasispecies with considerable genetic diversity. This study is to explore the selection of infectious ecosystems on dominant variants and their evolutionary dynamics of ALV-J between DF1 cells and specific-pathogen-free (SPF) chickens. High-throughput sequencing platforms provide an approach for detecting quasispecies diversity more fully.

Results: An average of about 20,000 valid reads were obtained from two variable regions of gp85 gene and LTR-U3 region from each sample in different infectious ecosystems. The top 10 dominant variants among ALV-J from chicken plasmas, DF1 cells and liver tumor were completely different from each other. Also there was a difference of shannon entropy and global selection pressure values $(\omega)$ in different infectious ecosystems. In the plasmas of two chickens, a large portion of quasispecies contained a 3-peptides "LSD" repeat insertion that was only less than $0.01 \%$ in DF1 cell culture supernatants. In parallel studies, the LTR-U3 region of ALV-J from the chicken plasmas demonstrated more variants with mutations in their transcription regulatory elements than those from DF1 cells.
\end{abstract}

Conclusions: Our data taken together suggest that the molecular epidemiology based on isolated ALV-J in cell culture may not represent the true evolution of virus in chicken flocks in the field. The biological significance of the "LSD" insert and mutations in LTR-U3 needs to be further studied.

Keywords: Subgroup J avian leukosis virus, Infectious ecosystem, 3-peptides LSD repeat insert (LSD ${ }^{+}$, Deep sequencing

\section{Background}

Avian leukosis virus (ALV) is an oncogenic retrovirus that induced lymphoid tumors in chickens and its genomic structure and molecular characteristics are well defined. It plays a critical role in the discoveries of reverse transcriptase, v-oncogenes and proto-oncogenes [1]. According to the host range, viral envelope interference and cross-neutralization patterns, avian leukosis viruses (ALVs) are classified into six subgroup (A to J) in chickens. ALV-J was first detected in meat-type chickens in the late 1980's [2], and then spread globally [3-8]. So far, ALV-J is

\footnotetext{
* Correspondence: zhaopeng@sdau.edu.cn; zzcui@sdau.edu.cn ${ }^{\dagger}$ Equal contributors

${ }^{1}$ College of Veterinary Medicine, Shandong Agricultural University, Taian 271018, China

Full list of author information is available at the end of the article
}

more pathogenic and mutate easily than other subgroups [9]. Although the eradication programs on ALV-J have been conducted in meat-type chickens since its discovery, it had spread into egg-type stock and the Chinese local breeds, which caused significant economic losses in China during the last 10 years $[10-15]$.

Proteins gp 85 and gp37 are encoded by the envelope gene of ALV, while gp85 protein constitute globular structures on the surface of the virus, which is closely associated with the process of viral binding and determine the specificity of subgroups. To understand molecular epidemiology of ALV-J among different types of chickens with various genetic backgrounds in many parts of the world, more than 200 ALV-J isolates have been subsequently sequenced and compared with gp 85 region of 
envelope gene since late 1980s [4, 10, 16-23]. The early study suggested that gp85 sequences of the ALV-J strains isolated from different geographical areas and farms in different years showed highly variable and their similarity varied in the range of $80-100 \%$. In terms of the $g p 85$ identity, the later isolates seemed to deviate gradually from the earliest isolate HPRS-103 [7]. However, many new isolates were obtained from different provinces of China after 1999. We found that no further deviate from HPRS-103 and all of their gp85 sequences still varied in the same range [4]. There was also no evidence to show further sequence deviation from HPRS-103 even for the ALV-J strains isolated in the recent 10 years from layers or Chinese local breeds of chickens [10,11, 13, 14, 24]. In addition, among 10 ALV-J isolates from ten individual layers with myelocytomas from the same flock demonstrated that they varied in the range of $80.3-97.1 \%$ in $g p 85$ region [25]. It seemed to suggest that there was no close relationship between ALV-J gp 85 homology levels and its pathogenicity or adaptation to different chicken breeds with different genetic backgrounds, although there were some epidemic phenomena indicated that ALV-J evolved to higher pathogenicity in different breeds of chickens.

In the past 30 years, almost all the molecular epidemiological data have been obtained by sequencing DNA fragments amplified and cloned from ALV-J infected CEF or DF1 cells. Such process would set up a bias for selection of certain quasispecies from the large population of viral particles in the given pathologic materials, for instance tumor tissues. By such selection, some significant variants associated with pathogenicity or adaptation to different genetic breeds may be survived by selective pressures. Wellehan reported that the dominant variants of San Miguel Sea Lion Virus populations altered significantly after its replication ecosystem switched from infected sea lions to cell cultures for 5 passages, the rare variants in sea lions became the dominant ones in cell cultures [26].

In this study, we analyzed and quantitatively compared dominant variants between ALV-J population replicated in infected chickens and cell cultures with the aid of deep sequencing-based method. The purpose of this study is to advance in understanding if cell culture ecosystem would cause selection pressures different from that of chickens with ALV-J infection, and whether the selection pressures would influence the evolution of ALV dominant variants. With these studies, we hope to identify specific epitopes or domains on $g p 85$, or other genes, such as in the region of $L T R-U 3$, which may associate with the differential selective pressures.

\section{Methods}

\section{Sample preparation}

The avian cell line DF-1 were obtained from the American Type Culture Collection (Manassas, VA, USA). These cells were grown in Dulbecco's modified Eagle's medium (DMEM; gibco, USA) supplemented with 10\% fetal bovine serum and $100 \mathrm{mg} / \mathrm{ml}$ of penicillin and streptomycin. A liver with myloid tumors was collected aseptically from clinically hy-line variety brown with spontaneous infections and identified as ALV-J via virus isolation (Genbank: KR049171, KR049172). Tumor homogenate prepared in plasma-free DMEM was lysed, the supernatant was purified by high-speed centrifugation and 0.22-um-pore-size cellulose-acetate filtration. The resultant purified tumor suspension was designated as original liver suspension (Ori) which was used as the viral strain in laboratory experiments, and the concentration was about 1500 $\mathrm{TCID}_{50} / 100 \mathrm{ul}$.

In vitro group, $3000 \mathrm{TCID}_{50}$ Ori were inoculated into DF-1 cells in logarithmic phase and maintained for 5 days as one passage. Then the infected DF1 cells were cultured via serial passages and cell free cultured supernatants were harvested at the 1st and 5th passage (P1 and P5). In vivo group, a total of 10 one-day-old specific pathogen free (SPF) chickens from the SPAFAS Co. (Jinan, China; a joint venture with Charles River Laboratory, Wilmington, MA, USA) were inoculated intraperitoneally with 3000 TCID $_{50}$ Ori. The blood plasma collections were performed for virus isolation, while antibodies of ALV-J were detected at 2, 4 and 6 weeks post inoculation, respectively. Following inoculation, plasma was obtained from whole blood and stored at $-80{ }^{\circ} \mathrm{C}$. Two plasma samples free of antibody at 2 weeks of sampling and the cell culture from the 1st and 5th passages were chosen for high throughput sequencing (C1 and $\mathrm{C} 2$ ). The animal infection protocol was reviewed and approved by the Shandong Province Animal Ethics Committee.

\section{RNA extraction, RT-PCR and sequencing}

Total viral RNA was extracted from samples of two ecosystems and original liver inoculum (Ori) using MagMAX Viral RNA isolation Kit (Life Technologies, USA) following the manufacturer's instructions. Each sample was amplified using a forward primer with a sixdigit error-correcting barcode as described earlier [27]. In addition, a 2-bp GT linker was added between the barcode and the $5^{\prime}$ end of the forward primer to avoid a potential match between the barcode and the target sequences. Therefore, the forward primer was barcode-GT-primer, in which the barcode indicates the six barcode sequences that are specific to different samples, then three pairs of primers were designed according to the reference sequence HPRS-103 (Genbank: Z46390), namely gp85-A, gp85-B and LTR-U3 (Additional file 1: Table S1 and Figure S1). ALV-specific RT-PCR targeting the hypervariable region of the gp85 and LTR-U3 genes were then performed on the viral RNA using the two-Step RT-PCR Kit (TAKARA, China) at $42{ }^{\circ} \mathrm{C}$ for $45 \mathrm{~min}, 5 \mathrm{~min}$ 
denaturation at $95{ }^{\circ} \mathrm{C}$, followed by 35 cycles of denaturation at $95{ }^{\circ} \mathrm{C}$ for $5 \mathrm{~min}, 95{ }^{\circ} \mathrm{C}$ for $30 \mathrm{~s}$; annealing at $53{ }^{\circ} \mathrm{C}$ for $30 \mathrm{~s}$, and extension at $72{ }^{\circ} \mathrm{C}$ for $30 \mathrm{~s}$, with a final extension step at $72{ }^{\circ} \mathrm{C}$ for $10 \mathrm{~min}$. The PCR products from both rounds were run of this reaction on a $1 \%$ agarose gels and scored. Bands of interest in the gels were cut out and the DNA was extracted from the gel using Qiagen Quick Gel Extraction Kit. The Products were quantified with a NanoDropND-1000 spectrophotometer (Thermo Fisher Scientific, Waltham, MA). A mixture of the amplicons was then used for sequencing on Illumina MiSeq platform according to the manufacturer's instructions at the Beijing Genomics Institute (Shenzhen, China). A base-calling pipeline (Sequencing Control Software, SCS; Illumina) was used to process the raw fluorescent images and the call sequences, and data quality assessment were performed on the MiSeq instrument.

\section{Data analysis}

Raw nucleotide sequences were filtered, aligned, trimmed and translated using pre-specified criteria applied uniformly. On average, there are $95 \%$ of the data above a quality value of Q30, which demonstrates a good quality of the demultiplexed reads. Then switch nucleic acid sequences of A, B fragment into amino acid sequences and threw away sequences with no biological significance for the following analysis (reads appeared more than 2 times were retained). The dominant gp85 and LTR-U3 variants of the samples were compared with each other under different infectious ecosystems using the Clustal W algorithm in MegAlign program of the DNASTAR package. Transcriptional regulatory elements in the U3 region were analyzed by the online service system of NSITE (Recognition of Regulatory motifs) of Soft Berry (http:// www.softberry.com/berry.phtml). The statistical analysis was done by Duncan's multiple range test.

In order to investigate quasispecies diversity under different ecosystems, we calculated the Shannon entrophy using clean reads of each sample.

Formula followed:

$$
\begin{aligned}
& H_{\text {shannon }}=-\sum_{i=1}^{S_{\text {obs }}} \frac{n_{i}}{N} \ln \frac{n_{i}}{N} \\
& \operatorname{var}\left(H_{\text {shannon }}\right)=\frac{\sum_{i=1}^{S_{\text {obs }}} \frac{n_{i}}{N}\left(\ln \frac{n_{i}}{N}\right)^{2}-H_{\text {shannon }}^{2}+\frac{S_{o b s}-1}{2 N^{2}}}{N}
\end{aligned}
$$

$S_{\text {obs }}=$ The amount of haplotype observed by sequencing

$n_{i}=$ Number of sequences for haplotype $i$

$N=$ The total valuable sequence number obtained by sequencing

To minimize potential sampling bias and reduce the computation load, we performed a bootstrapping strategy for the clean reads of each sample. For each re-sampling with replacement, phylogenetic analysis was performed using RaxML [28] with 200 bootstrap replicates, under the GAMMACAT substitution model. All other parameters were set to their default values. Global selection pressure values $(\omega)$ were estimated using HyPhy method [29].

\section{Results}

\section{MiSeq high throughput sequencing data}

After several filtering steps, about 94 to $97 \%$ of the nucleic acid sequences (LTR-U3) or $86-93 \%$ of the amino acid sequences (gp85-A and gp85-B) from any sample of the raw reads were retained for subsequent analyses. The raw reads and the filtered reads obtained using MiSeq High-throughput Sequencing of the extracted RNA generated a median of more than 20,000 reads per sample (Additional file 1: Table S2).

\section{Comparison of the ratios of haplotypes in different viral infectious ecosystems}

The ratios of sequence haplotypes to total valid reads for gp85-A, gp85-B and LTR-U3 fragments from both plasmas of two chickens (C1 and C2) and DF1 cell culture supernatants of two different passages (P1 and P5) were decreased significantly as compared to that of the original liver inoculum (Ori) (Additional file 1: Table S3). The results suggested there might be some selective pressures on quasispecies of both gp85-A (hr1 and vr2 regions), gp85-B (hr2 and vr3 regions) and LTR-U3 fragments when ALV-J from the Ori replicated in chickens or in DF1 cell cultures. Some variants in Ori were decreased dramatically to undetectable levels when replication ecosystem changed.

\section{Evolutionary dynamics of gp85-B (hr2 and vr3) under different infectious ecosystems}

The dominant variants of gp85-B altered dramatically after replication under the two different ecosystems. The percentage of the most dominant variant of gp85-B in Ori decreased to a very low level and even became undetectable in infected chickens or cell culture supernatants, while some other sub-dominant variants were increased and decreased at a high and low percentages (Fig. 1a). Actually, the top 5 dominant variants in chicken plasmas or cell cultures were rare ones in Ori (Fig. 1b). It suggested that there were some strong selective pressures having influence on the evolution of dominant variants of gp85-B from infectious ecosystems.

The most dominant variant (BO0001) accounting for $32.85 \%$ in the Ori did not appear among the first 10 dominant quasipecies in either cell culture supernatants or chicken plasmas. We also find that there are two identical variants within the first 10 dominant variants of gp85-B from both chicken samples (C1 and C2). Sequences of the first dominant variant (BC1001) from $\mathrm{C} 1$ 


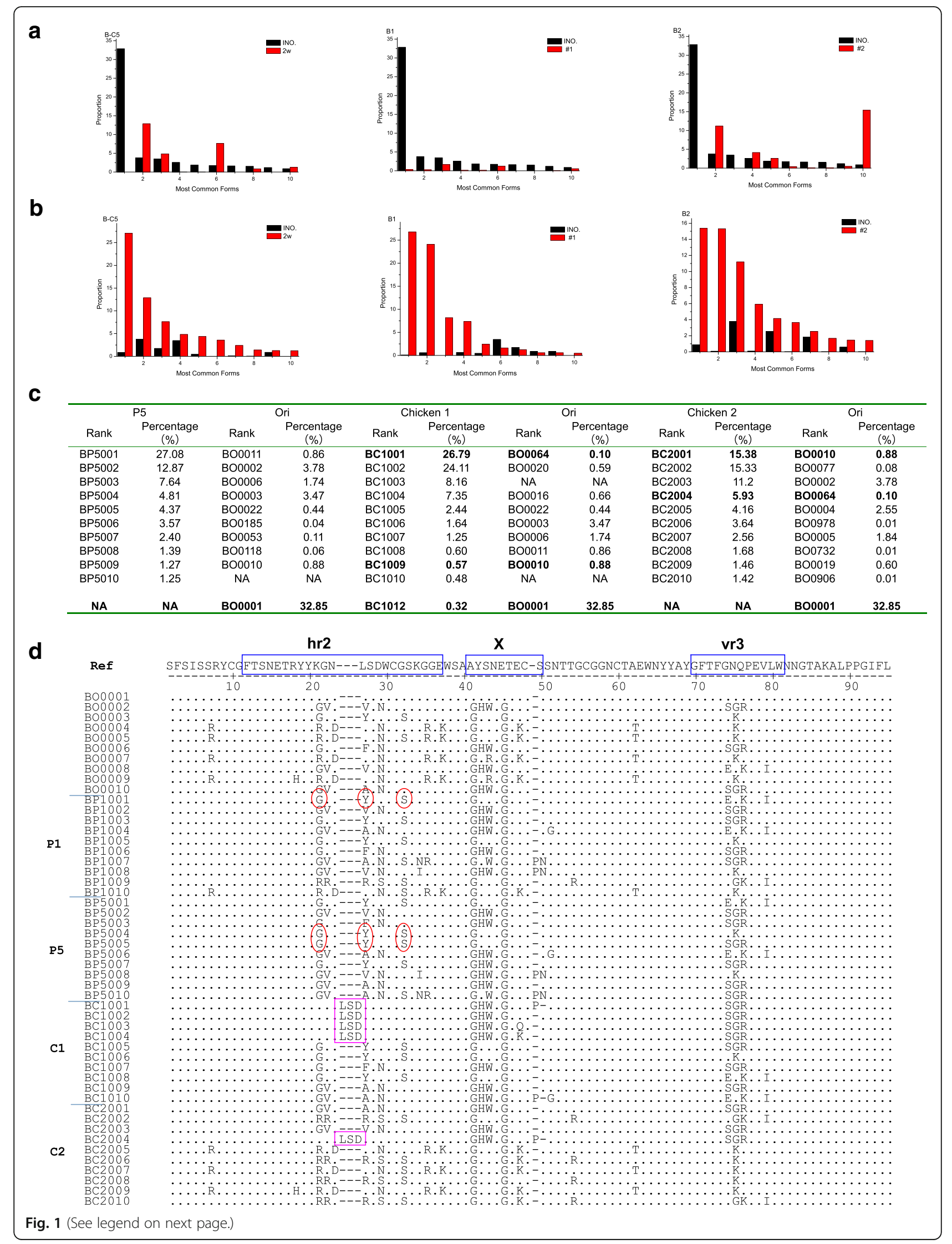




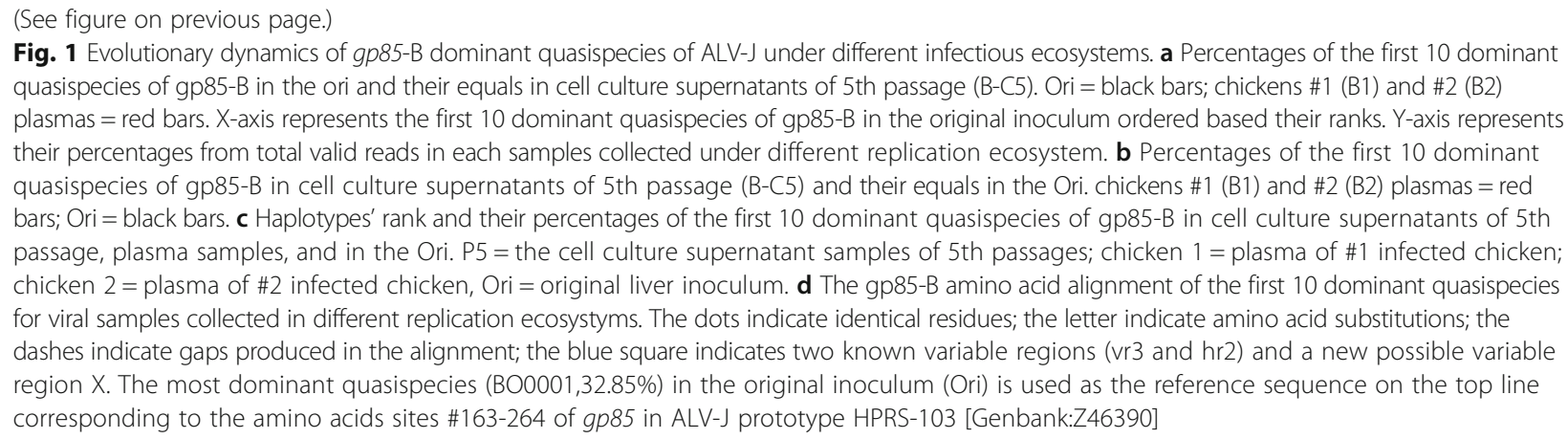

and the fourth dominant variant (BC2004) from $\mathrm{C} 2$ are $100 \%$ identical to the $64^{\text {th }}$ variant (BO0064) in the Ori. Similarly, the most dominant variant (BC2001) from C2 and the $9^{\text {th }}$ dominant variant $(\mathrm{BC} 1009)$ from $\mathrm{C} 1$ are $100 \%$ identical to the $10^{\text {th }}$ dominant variant (BO0010) in the Ori (Fig. 1c). Although the other 8 dominant variants are different between the two chicken samples, but they have high homologies and only limited number of different sites than those from cell culture supernatants (Fig. 1d).

\section{Characterization of a specific domain of $g p 85-\mathrm{B}$ associated with selective evolution of dominant variants under different viral replication conditions}

The differences of amino acids in $g p 85$-B dominant variants under different viral replication conditions are mainly in two known variable regions hr2 (aa\#11-36) and vr3 (aa\#69-80), or a new possible variable region $\mathrm{X}$ (aa\#40-48). The most prominent difference is that the major dominant variants from chicken plasmas have a 3-peptides LSD repeat insert $\left(\mathrm{LSD}^{+}\right)$when compared to samples from the Ori or cell culture supernatants. Although the evolutionary dynamics of $\mathrm{LSD}^{+}$in two chickens are not the same. In the plasma of chicken \#1 (C1), all the top 4 dominant variants (BC1001, BCC1002, BC1003, BC1004) accounting for $66.32 \%$ from total valid reads get $\mathrm{LSD}^{+}$at $2 \mathrm{w}$ post infection which is significantly increased compared to these equals in Ori consisting of only $1.35 \%$. In another chicken $(\mathrm{C} 2)$, only one as the $4^{\text {th }}$ dominant variant $(\mathrm{BC} 1004)$ with $\mathrm{LSD}^{+}$appears in the top 10 dominant variants, which accounting for 5.93\% from the total valid reads, that was still a significant increase as the identical sequence haplotype (BO0064) in Ori was only $0.10 \%$. In contrast, there is no $\mathrm{LSD}^{+}$in the top 10 dominant variants in cell culture, and only 4 haplotypes with $\mathrm{LSD}^{+}$ consisted of only $0.02 \%$ in its total 29,986 valid reads are found among 3846 variants haplotypes, which was dramatically declined from $1.35 \%$ when compared to that only 163 haplotypes from 4047 variants haplotypes in Ori.

All variants with $\mathrm{LSD}^{+}$in different ecosystems were compared and analyzed. In $\mathrm{C} 1$, the top $10 \mathrm{LSD}^{+}$dominant variants accounting for $66.95 \%$ of the total valid reads, compared to only $1.45 \%$ in the Ori. While in $\mathrm{C} 2$, the first 10 dominant variants with $\mathrm{LSD}^{+}$consisted of $6.62 \%$ of the total valid reads, but only $0.70 \%$ in the Ori. It indicates that variants with $\mathrm{LSD}^{+}$were dramatically increased by positive selection after replication in chickens. Specifically, there are three completely identical variants with $\mathrm{LSD}^{+}$in two chicken plasma samples (BC1001 vs $\mathrm{BC} 2004, \mathrm{BC} 1002$ vs $\mathrm{BC} 2022$, and $\mathrm{BC} 1022$ vs $\mathrm{BC} 2040$ ). However, evolution of variants with $\mathrm{LSD}^{+}$are to the opposite direction after replication in DF1 cell cultures, that is, percentages of $\mathrm{LSD}^{+}$positive variants decline rapidly and even disappear. Some $\mathrm{LSD}^{+}$positive variants detected in infected chicken plasma are not detectable in the Ori even all reads are analyzed (Additional file 1: Table S4). There are some amino acid alterations in $\mathrm{x}$ and $\mathrm{vr} 3$ regions, but such variations trend to be convergent (Additional file 1: Figure S2). Also two pairs of LSD ${ }^{+}$ positive and $\mathrm{LSD}^{+}$negative variants are compared for their antigenic index by computational analysis. The results indicated that $\mathrm{LSD}^{+}$significantly increased the antigenic index in the new domain around the LSD insert (Fig. 2).

\section{Mutational analysis of $g p 85-B$ under different infectious ecosystems}

By analyzing the data from deep sequencing, mutational frequency of each site in the $\mathrm{LSD}^{+}$domain is also compared independently among gp 85 -B quasispecies of ALV-J replicated in different ecosystems (Fig. 3). Each amino acid of L, S and D at the insertion sites (aa\#23-\#25) appears at the frequency of $2.78-2.79 \%$ in Ori, but it dramatically decreases to $0-0.01 \%$ after ALV-J is passaged in DF1 cells. In contrast, their frequencies were increased to $85.31-85.34 \%$ and $8.58-8.60 \%$ respectively in $\mathrm{C} 1$ and $\mathrm{C} 2$ which were very close to the frequency of 85.31 and $8.58 \%$ of the entire "LSD", suggesting that the positive effect in infected chickens and negative selection in cell cultures were mainly associated with the intact 3-peptides insertion of LSD.

Besides, specific mutations of another 3 important sites in $g p 85$-B were recognized to be associated with positive 


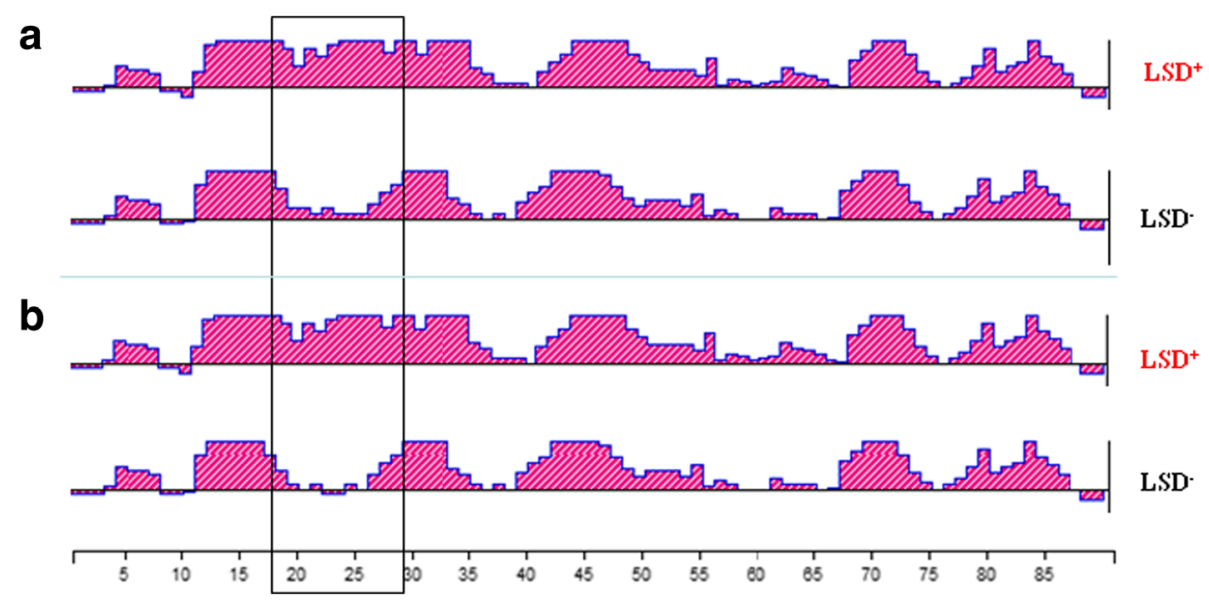

Fig. 2 Comparison of the antigen index between the gp85-B quasispecies with or without $\mathrm{LSD}^{+}$. a The comparison of antigen index between $102001\left(\mathrm{LSD}^{+}\right)$and B102007 (LSD-). b The comparison of antigen index between B202004 (LSD ${ }^{+}$) and B202003 (LSD). Antigenic profiles calculated with Jameson and Wolf (Jameson and Wolf, 1988) algorithm from the linear amino acid sequences, the different area of antigen index with or without LSD insertion mutation was marked with a black frame

selective pressures in DF1 cells. The proportions of variants consisted of the 3 none-successive amino acids G-Y-S at aa positions \#20, \#26 and \#30 were significantly increased, to $90.34,49.32$ and $60.12 \%$ in P1 and 99.36, 53.71 and $55.84 \%$ in P5 from $30.18,11.02$ and $17.08 \%$ in Ori, respectively (Fig. 3). In contrast, the proportions were 12.21, 7.33 and $8.3 \%$ in $\mathrm{C} 1$ and $39.51,0.18$ and $39.39 \%$ in $\mathrm{C} 2$. Further analysis of sequence data in Fig. 1 indicated that the $1^{\text {st }}, 4^{\text {th }}, 5^{\text {th }}$ and $7^{\text {th }}$ ones among the top 10 dominant variants in $5^{\text {th }}$ passage cell culture contained the none-

\begin{tabular}{|c|c|c|c|c|c|c|}
\hline Position & 20 & 26 & 31 & 23 & 24 & 25 \\
\hline \multirow[t]{2}{*}{ Reference } & & & & & & \\
\hline & K54.80 & L.67.43 & G82.77 & $ـ_{97.21}$ & _97.21 & ${ }_{-97.21}$ \\
\hline \multirow[t]{3}{*}{ Ori } & G30.38 & Y11.22 & S17.08 & L2.78 & S2.28 & D2.79 \\
\hline & R14.50 & V10.88 & D0.15 & P0.01 & P0.01 & \\
\hline & G90.34 & Y 49.32 & S60.12 & _99.99 & _99.99 & _99.99 \\
\hline \multirow[t]{3}{*}{ P1 } & R9.14 & A16.36 & G39.81 & & & \\
\hline & $\_.45$ & V14.59 & N0.05 & & & \\
\hline & G99.36 & Y53.71 & S55.84 & _99.99 & _99.99 & _ 99.99 \\
\hline \multirow[t]{3}{*}{ P5 } & R0.44 & V20.72 & G44.07 & L0.01 & S0.01 & D0.01 \\
\hline & _- 0.11 & F14.13 & N0.03 & & & \\
\hline & K86.24 & L86.99 & G91.62 & L85.32 & S85.34 & D85.31 \\
\hline \multirow[t]{3}{*}{ C1 } & G12.21 & Y7.33 & S8.30 & _14.52 & ${ }_{-}^{14.52}$ & ${ }_{-}^{14.52}$ \\
\hline & R1.44 & A2.26 & D0.06 & P0.11 & P0.10 & G0.11 \\
\hline & R51.74 & R34.16 & G60.55 & _91.39 & _91.39 & _91.38 \\
\hline \multirow[t]{2}{*}{$\mathrm{C} 2$} & G39.53 & L26.16 & S39.39 & L8.58 & S8.60 & D8.58 \\
\hline & K8.64 & A21.48 & N0.03 & P0.01 & & G0.01 \\
\hline \multicolumn{7}{|c|}{$\begin{array}{l}\text { Fig. } 3 \text { Comparison of amino acid changes in six sites on } 9 p 85-B \\
\text { under different infectious ecosystems. The first } 3 \text { frequent amino } \\
\text { acids at each site and their percentages were listed for samples } \\
\text { collected from the original inoculum. } P 1=\text { the } 1^{\text {st }} \text { passage cell } \\
\text { culture; } P 5=\text { the } 5^{\text {th }} \text { passage cell culture; } \# 1=\text { chicken } 1 ; \# 2=\text { chicken } 2 \text {. } \\
\text { The capital letters indicate specific amino acid. The numerical numbers } \\
\text { indicate the sites of the gp } 85-B \text { by use of the most dominant quasispecies } \\
\text { in the original inoculum as the reference }\end{array}$} \\
\hline
\end{tabular}

successive G-Y-S and consisted $38.96 \%$ of total reads, while two of the first 10 dominant variants in the original liver inoculum contained the G-Y-S which accounting for only $5.21 \%$ of total valid reads.

\section{Comparisons of the Shannon entropy and global selection pressure values $(\omega)$ under different infectious ecosystems} To roughly quantify the pressures that quasispesis underwent under different infectious ecosystems, we calculated the Shannon entropy and the global selection pressure values $(\omega)$. Our results showed the Shannon entropy in Ori was the highest, but when inoculated into chickens or DF1 cells different degree of decline were observed (Table 1). On the global selection pressure values $(\omega)$, those in cells were relatively stable at about 0.61 , bigger than that from the Ori, but there were two different situations in the chickens ecosystem. Specifically, the $\omega$ values of the quasispecies in $\mathrm{C} 1$ were higher than those in P1 and P5, but the Shannon entropy is lower. Moreover, the $\omega$ values of the quasispecies in $\mathrm{C} 2$ were lower than those in P1 and P5, however the Shannon entropy is higher.

Table 1 The Shannon entropy and global selection pressure values $(\omega)$ under different infectious ecosystems

\begin{tabular}{lll}
\hline Ecosystems & The Shannon entropy & Global selection pressure values $(\omega)$ \\
\hline Ori & 4.90 & $0.59 \pm 0.03^{\mathrm{A}}$ \\
C1 & 3.65 & $0.63 \pm 0.04^{\mathrm{B}}$ \\
C2 & 4.52 & $0.54 \pm 0.04^{\mathrm{C}}$ \\
P1 & 4.40 & $0.61 \pm 0.04^{\mathrm{D}}$ \\
P5 & 4.24 & $0.61 \pm 0.04^{\mathrm{D}}$ \\
\hline
\end{tabular}

Each value are calculated by 200 bootstrap re-samples of the distribution of variants. Each column in the upper label with different letters mean significant difference on Duncan's multiple range test $(P<0.01)$ 


\section{Comparisons of dominant variants in LTR-U3 region under different infectious ecosystems}

The high throughput sequencing of LTR-U3 region showed that the most dominant variants had not changed in both chicken plasmas and DF1 cell culture supernatants when compared to the Ori. However, the other subdominant variants of $U 3$ region evolved into different directions under different infectious ecosystems, in vivo and cell cultures. There were 7 of the top 10 variants were exactly the same in two chicken plasma samples which consisted of 70.08 and $72.62 \%$ of the total valid reads in the two chickens respectively (Fig. 4a). But all the left 9 major dominant variants of $U 3$ in cell cultures were completely different from those of chicken plasma.

Sequence alignment analysis demonstrated that $U 3$ region was more conservative in ALV-J replicated in cell cultures than in infected chickens, as the other 9 major sub-dominant variants haplotypes from DF1 cell culture supernatants had fewer mutations than that from 2 chicken plasma samples when compared to the most dominant variant common in different infectious ecosystems. More importantly, the most base alterations were located within some motifs as transcription regulatory elements (Fig. 4b) in samples from two chickens and the

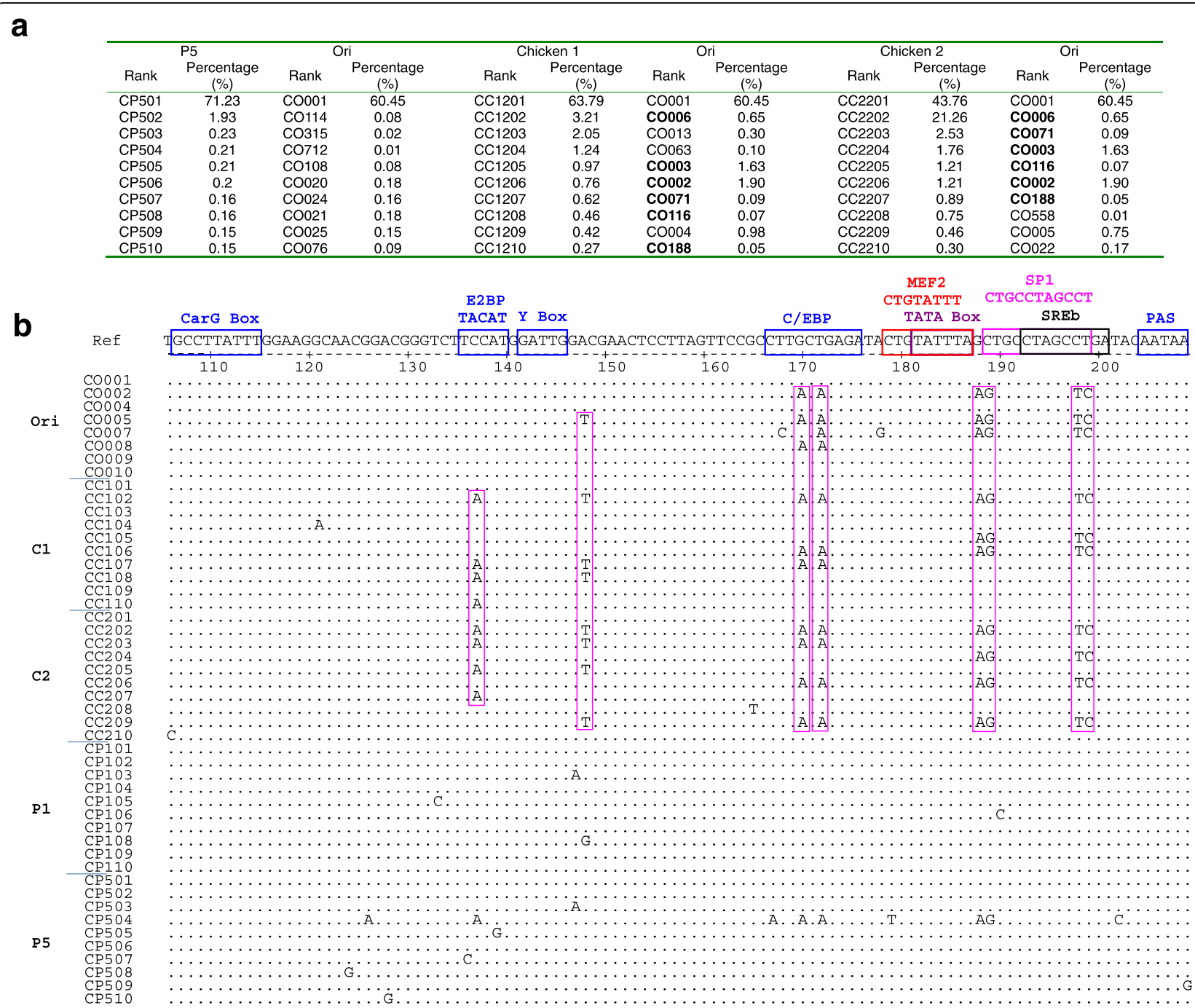

Fig. 4 Evolutionary dynamics of the first 10 dominant quansispecies of U3 fragment in different infectious ecosystems. a The first 10 dominant quasispecies haplotype (in the order according ranks) and their percentages of total valid reads in DF1 cell cultures (combined two passages, the left part). $\mathrm{CC1001}=$ the quasispecies ranked the first in segment $\mathrm{C}$ from chicken 1; $\mathrm{O} 0=$ the original liver inoculum; $\mathrm{C} 2=$ chicken 2; $\mathrm{P} 1=$ passage ; P5 = passage 5 in cell culture. The last 3 numbers represents their ranks in the quasispecies population in each sample. $\mathbf{b}$ Base sequence alignment of the first 10 dominant quasispecies of $\mathrm{U} 3$ detected in each different infectious ecosystems. The reference sequence is the most dominant quasispecies in the original inoculum $(C O 0001,60.45 \%)$, corresponding to bases \#122-225 of LTR of ALV-J prototype strain HPRS-103. The dots indicate identical residues, while the letters indicate amino acid substitutions. The motifs as transcription regulatory elements were labeled on the top 
Ori. There were eight common mutation sites and similar replacement in the ecosystems of the two chickens, and then C/EBP, SP1, MEF2 and SREb regulatory elements losing their integrity but made one more motif of E2BP due to the replacement of $\mathrm{C}$ to $\mathrm{A}$ at the site \#137. Obviously, it is due to differences of infectious ecosystems.

\section{Discussion}

The object of this study was to understand if there is any influence from different infectious ecosystems on ALV-J dominant variants evolution. Both chickens and DF1 cell cultures were inoculated with the same Ori. The RT-PCR products of each sample were directly sequenced and analyzed by the deep sequencing, it produced extreme large sequence data covering genome variants even at very low frequencies. With the technology progresses on diversity in quasispecies and its evolutionary dynamics were obtained under different immunoselective pressures, antiviral drugs, and various viruses, such as HIV [30-33], and hepatitis B, C, and E viruses [34,35] and some animal viruses [36].

Deep Sequencing generated a median of more than 20,000 reads per sample, which were large enough to compare and understand the quasispecies diversity from these samples. The ratios of haplotypes/valid reads of 3 fragments from chickens and cell culture samples were decreased compared to the Ori, suggesting that both infectious ecosystems demonstrated a negative selective effect on some quasispecies in the Ori. Among the 3 fragments sequenced, gp85-A fragment was less influenced by selective pressures, but gp $85-\mathrm{B}$ fragment was significantly influenced. Some dominant variants in the Ori was dramatically decreased in the inoculated chicken plasma and cell culture supernatant samples, but some very rare variants became the dominant ones. The results provided the direct experimental evidence that the infectious ecosystems would dramatically influenced the evolution of viral quasispecies. It is clear that the Ori was liver suspension and its ALV-J quasispecies mainly replicated in liverassociated cells, however, viruses in chicken plasmas or cell culture supernatant came from all kinds of sensitive cells in the body or replicated only in DF1 cells after the Ori was infected chickens or cell cultures. Bioinformatic analysis results showed the Shannon entropy in Ori was the highest, but when inoculated into chickens or DF1 cells different degree of decline were observed, which indicated there were some pressures in the ecosystems. On the global selection pressure values $(\omega)$, there was a big individual difference in chickens, but relatively stable in DF1 cells. There was significant difference $(P<0.01)$ between the two groups from chickens and DF1 cells, which also suggested different selective pressure in the two groups.
The envelope protein gp85 is related to recognition and adhesion to sensitive cells, and also is the major antigen for viral neutralization [9]. The diversity in $g p 85$ sequence, especially epitopes at certain sites may influence the tropism of virus quasispecies to different types of cells in chicken body. For example, it has been reported that ALV-J prototype HPRS-103 has a low tropism for bursal follicles cells but does replicate well in cultured blood monocytes [9]. The most interesting result in this study is the discovery of the 3-peptides "LSD" repeat insertion $\left(\mathrm{LSD}^{+}\right)$in novel dominant variants of gp85-B fragments emerging in chicken plasmas samples, which increased the antigen index in the sub-region. However, there was no $\mathrm{LSD}^{+}$positive variants among the top 10 dominant variants in two DF1 supernatant samples. Referencing to the principle of site-by-site positive selection analysis using the two rate fixed-effects likelihood (FEL) method [37], after artificial calculation we found that the $\mathrm{LSD}^{+}$were under positive selection in chickens, while negative selection in DF1 cells. It might help to explain how evolution of different variants with specific epitope could be influenced by some selective pressures from ecosystem or its infectious ecosystems such as different organs, tissues or cell types. We speculate that variation in gp85 sequence similarity may not necessarily reflect its relationship to evolution in terms of higher pathogenicity to different genetic breeds of chickens, but some specific epitopes or domains on gp85 would influence.

LTR- $U 3$ region of ALV has only about 250 bp but contains several biological active motifs and enhancers influencing transcription and virus replication [38, 39], also it is a fragment easy to mutate on the ALV genome. However, analysis of deep sequencing data of $U 3$ region in different samples demonstrated that the viral population in chicken plasma samples came from ALV-J replicated in different types of cells, organs and tissues of the chicken and experienced quite different ecosystem selection pressures. Chicken plasma samples and DF1 supernatant sample had the same most dominant variants of $U 3$ as the Ori, which indicated that different infectious ecosystems did not have as high selective pressures on the evolution of $U 3$ quasispecies as that of $g p 85-\mathrm{B}$. But in the two chicken plasmas there were 6 absolutely identical variants for the top 10 sub-advantage variants while no reapeat with the top 10 sub-dominant variants in cell culture. Although the first 10 dominant $U 3$ variants were very conservative in cell culture supernatants, several sub-dominant variants from chicken plasma samples and Ori had mutations in its regulation elements CIEBP, SP1, MEF2 and SREb. Concerning the biological significance, its needs to be further investigated and studied.

\section{Conclusions}

In conclusion, this study is the first to explore the replication of ALV-J in different ecosystem using deep 
sequencing technique. We found that significant differences in dominant variants and their evolution dynamics of gp85 from ALV-J in infected chickens or cell cultures. Especially, a tri-peptides "LSD" insert associated with positive selective pressures in infected chickens and negative selective pressures in DF1 cell cultures in gp85 were identified. It suggests that the replication ecosystem has a significant influence on the evolution of viruses. The molecular epidemiology studies based on the isolated ALV-J in cell culture may not represent the true evolution of these viruses in infected chicken flocks in the field.

\section{Additional file}

Additional file 1: Table S1. Three pairs of primers for Miseq Highthroughput Sequencing. Table $\mathbf{S} 2$ The numbers of raw reads and clean reads in each sample. Table $\mathbf{S} \mathbf{3}$ Variations of ratios of sequence haplotype numbers to total valid reads in different replication ecosystems. Table $\mathbf{S 4}$ Dynamics of the first $10 \mathrm{LSD}+$ positive quasispecies of gp85-B in different replication ecosystems. Figure S1 The structure of env and LTR in ALV-J. Figure S2 Amino acid alignment of top 10 LSD+ positive quasispecies of gp85-B in different replication ecosystems. (DOC $311 \mathrm{~kb}$ )

\section{Abbreviations}

$L^{2} D^{+}$: A 3-peptides LSD repeat insert; ALV-J: Avian leukosis virus subgroup J; $\mathrm{P} 1$ and P4: The $1^{\text {st }}$ and $5^{\text {th }}$ passage; $\mathrm{C} 1$ and C2: Chicken 1 and chicken 2; Ori: Original liver suspension; SPF: Specific pathogen free

\section{Acknowledgements}

We thank Lucy F. Lee for helpful discussions. We also thank BGI-Shenzhen for Miseq sequencing.

\section{Funding}

This work was supported by grants from the National Natural Science Foundations of China (grant numbers: 31472216,31402226$)$ and the Natural Science Fundation of Jiangsu Province (grant No.BK20151317).

\section{Availability of data and materials}

All the data supporting the results are included in the article.

\section{Authors' contributions}

All authors approved the manuscript. MFF, DX, HT, CS, FJH, ZP and CZZ contributed to study design and data interpretation. MFF was the principal investigator. MFF and DX wrote the manuscript and produced all figures. All authors read, corrected, and approved the final manuscript prior to submission.

\section{Competing interests}

The authors declare that they have no competing interests.

\section{Consent for publication}

Not applicable.

\section{Ethics approval and consent to participate}

Ethical and humane treatment of animals used in this study was proposed to and approved by Shandong Province Animal Ethics Committee.

\section{Author details}

'College of Veterinary Medicine, Shandong Agricultural University, Taian 271018, China. ${ }^{2}$ Institute of Pathogen Biology, Taishan Medical College, Taian, Shandong, China. ${ }^{3}$ Poultry Institute, Chinese Academy of Agricultural Sciences, Yangzhou, Jiangsu, China.

Received: 10 May 2016 Accepted: 29 November 2016 Published online: 19 December 2016

\section{References}

1. Weiss RA, Vogt PK. 100 years of Rous sarcoma virus. J Exp Med. 2011;208:2351-5.

2. Payne LN, Brown SR, Bumstead N, Howes K, Frazier JA, et al. A novel subgroup of exogenous avian leukosis virus in chickens. J Gen Virol. 1991;72:801-7.

3. Bagust TJ, Fenton SP, Reddy MR. Detection of subgroup J avian leukosis virus infection in Australian meat-type chickens. Aust Vet J. 2004:82:701-6.

4. Cui Z, Du Y, Zhang Z, Silva RF. Comparison of Chinese field strains of avian leukosis subgroup J viruses with prototype strain HPRS-103 and United States strains. Avian Dis. 2003:47:1321-30.

5. Fadly AM, Smith EJ. Isolation and some characteristics of a subgroup J-like avian leukosis virus associated with myeloid leukosis in meat-type chickens in the United States. Avian Dis. 1999;43:391-400.

6. Nakamura K, Ogiso M, Tsukamoto K, Hamazaki N, Hihara H, et al. Lesions of bone and bone marrow in myeloid leukosis occurring naturally in adult broiler breeders. Avian Dis. 2000:44:215-21.

7. Silva RF, Fadly AM, Hunt HD. Hypervariability in the envelope genes of subgroup J avian leukosis viruses obtained from different farms in the United States. Virology. 2000;272:106-11.

8. Zavala G, Cheng S, Jackwood MW. Molecular epidemiology of avian leukosis virus subgroup $\mathrm{J}$ and evolutionary history of its $3^{\prime}$ untranslated region. Avian Dis. 2007:51:942-53.

9. Payne $L N$, Nair $V$. The long view: 40 years of avian leukosis research. Avian Pathol. 2012:41:11-9.

10. Dong X, Zhao P, Li W, Chang S, Li J, et al. Diagnosis and sequence analysis of avian leukosis virus subgroup J isolated from Chinese Partridge Shank chickens. Poult Sci. 2015;94(4):668-72.

11. Gao Y, Guan X, Liu Y, Li X, Yun B, et al. An avian leukosis virus subgroup J isolate with a Rous sarcoma virus-like 5'-LTR shows enhanced replication capability. J Gen Virol. 2015;96:150-8.

12. Gao $\mathrm{YL}$, Qin $\mathrm{LT}$, Pan W, Wang YQ, Le Qi X, et al. Avian leukosis virus subgroup J in layer chickens. China Emerg Infect Dis. 2010;16:1637-8.

13. Li Y, Liu X, Liu H, Xu C, Liao Y, et al. Isolation, identification, and phylogenetic analysis of two avian leukosis virus subgroup I strains associated with hemangioma and myeloid leukosis. Vet Microbiol. 2013;166:356-64.

14. Sun S, Cui Z. Epidemiological and pathological studies of subgroup J avian leukosis virus infections in Chinese local "yellow" chickens. Avian Pathol. 2007:36:221-6

15. Xu B, Dong W, Yu C, He Z, Lv Y, et al. Occurrence of avian leukosis virus subgroup J in commercial layer flocks in China. Avian Pathol. 2004;33:13-7.

16. Du Y, Cui ZZ, Qin AJ, Silva RF, Lee LF. Isolation of subgroup J avianleukosis viruses and their partial sequence comparison. Chinese Journal of Virology. 2000:16:341-6.

17. Gao Y, Yun B, Qin L, Pan W, Qu Y, et al. Molecular epidemiology of avian leukosis virus subgroup J in layer flocks in China. J Clin Microbiol. 2012;50:953-60.

18. Jiang L, Zeng X, Hua Y, Gao Q, Fan Z, et al. Genetic diversity and phylogenetic analysis of glycoprotein gp85 of avian leukosis virus subgroup J wild-bird isolates from Northeast China. Arch Virol. 2014;159:1821-6.

19. Lai H, Zhang H, Ning Z, Chen R, Zhang W, et al. Isolation and characterization of emerging subgroup $\mathrm{J}$ avian leukosis virus associated with hemangioma in egg-type chickens. Vet Microbiol. 2011;151:275-83.

20. Li H, Xue C, Ji J, Chang S, Shang H, et al. Complete genome sequence of a J subgroup avian leukosis virus isolated from local commercial broilers. J Virol. 2012;86:11937-8

21. Mao Y, Li W, Dong X, Liu J, Zhao P. Different quasispecies with great mutations hide in the same subgroup J field strain of avian leukosis virus. Sci China Life Sci. 2013;56:414-20.

22. Pan W, Gao Y, Qin L, Ni W, Liu Z, et al. Genetic diversity and phylogenetic analysis of glycoprotein GP85 of ALV-J isolates from Mainland China between 1999 and 2010: coexistence of two extremely different subgroups in layers. Vet Microbiol. 2012;156:205-12.

23. Wang $\mathrm{H}$, Cui Z. The identification and sequence analysis of ALV-J isolated from layers. Chin J Virol. 2008;24:369-75.

24. Wang Z, Cui Z. Evolution of gp85 gene of subgroup J avian leukosis virus under the selective pressure of antibodies. Sci China C Life Sci. 2006;49:227-34.

25. Bian XM, Li DQ, Zhao P, Cui ZZ. Continuous observation of subgroup J avian leukosis for three groups of commercial layer chicken. Sci Agric Sin. 2013;46:409-16.

26. Wellehan Jr JF, Yu F, Venn-Watson SK, Jensen ED, Smith CR, et al. Characterization of San Miguel sea lion virus populations using pyrosequencing-based methods. Infect Genet Evol. 2010;10:254-60. 
27. Hamady M, Walker JJ, Harris JK, Gold NJ, Knight R. Error-correcting barcoded primers for pyrosequencing hundreds of samples in multiplex. Nat Methods. 2008:5:235-7.

28. Stamatakis A, Ludwig T, Meier H. RAxML-III: a fast program for maximum likelihood-based inference of large phylogenetic trees. Bioinformatics. 2005;21:456-63.

29. Pond SL, Frost SD, Muse SV. HyPhy: hypothesis testing using phylogenies. Bioinformatics. 2005;21:676-9.

30. Bansode V, McCormack GP, Crampin AC, Ngwira B, Shrestha RK, et al. Characterizing the emergence and persistence of drug resistant mutations in HIV-1 subtype C infections using 454 ultra deep pyrosequencing. BMC Infect Dis. 2013;13:52.

31. Henn MR, Boutwell CL, Charlebois P, Lennon NJ, Power KA, et al. Whole genome deep sequencing of HIV-1 reveals the impact of early minor variants upon immune recognition during acute infection. PLoS Pathog. 2012;8:e1002529.

32. Recordon-Pinson P, Raymond S, Bellecave P, Marcelin AG, Soulie C, et al. HIV-1 dynamics and coreceptor usage in Maraviroc-treated patients with ongoing replication. Antimicrob Agents Chemother. 2013;57:930-5.

33. Rozera G, Abbate I, Ciccozzi M, Lo Presti A, Bruselles A, et al. Ultra-deep sequencing reveals hidden HIV-1 minority lineages and shifts of viral population between the main cellular reservoirs of the infection after therapy interruption. J Med Virol. 2012;84:839-44.

34. Rodriguez-Frias F, Tabernero D, Quer J, Esteban JI, Ortega I, et al. Ultra-deep pyrosequencing detects conserved genomic sites and quantifies linkage of drug-resistant amino acid changes in the hepatitis B virus genome. PLoS One. 2012;7:e37874.

35. Miura M, Maekawa S, Takano S, Komatsu N, Tatsumi A, et al. Deep-sequencing analysis of the association between the quasispecies nature of the hepatitis $C$ virus core region and disease progression. J Virol. 2013;87:12541-51.

36. Borucki MK, Chen-Harris H, Lao V, Vanier G, Wadford DA, et al. Ultra-deep sequencing of intra-host rabies virus populations during cross-species transmission. PLoS Negl Trop Dis. 2013;7:e2555.

37. Kosakovsky Pond SL, Frost SD. Not so different after all: a comparison of methods for detecting amino acid sites under selection. Mol Biol Evol. 2005;22:1208-22.

38. Ryden TA, Beemon K. Avian retroviral long terminal repeats bind CCAAT/ enhancer-binding protein. Mol Cell Biol. 1989;9:1155-64.

39. Zachow KR, Conklin KF. CArG, CCAAT, and CCAAT-like protein binding sites in avian retrovirus long terminal repeat enhancers. J Virol. 1992;66:1959-70.

\section{Submit your next manuscript to BioMed Central and we will help you at every step:}

- We accept pre-submission inquiries

- Our selector tool helps you to find the most relevant journal

- We provide round the clock customer support

- Convenient online submission

- Thorough peer review

- Inclusion in PubMed and all major indexing services

- Maximum visibility for your research

Submit your manuscript at www.biomedcentral.com/submit 